\title{
Postoperative Outcome in Children Aged between 6 and 10 Years in Major Abdominal Surgery, Neurosurgery and Orthopedic Surgery
}

\author{
Claudine Kumba (1) \\ Department of Pediatric Anesthesia and Critical Care, Necker Enfants Malades University Hospital, Assistance Publique \\ Hôpitaux de Paris, APHP, University of Paris, Paris, France \\ Email: claudine.kumba@gmail.com, claudine.kumba@aphp.fr
}

How to cite this paper: Kumba, C. (2021) Postoperative Outcome in Children Aged between 6 and 10 Years in Major Abdominal Surgery, Neurosurgery and Orthopedic Surgery. Open Journal of Pediatrics, $11,636-645$.

https://doi.org/10.4236/ojped.2021.114059

Received: October 9, 2021

Accepted: November 14, 2021

Published: November 17, 2021

Copyright $\odot 2021$ by author(s) and Scientific Research Publishing Inc. This work is licensed under the Creative Commons Attribution International License (CC BY 4.0).

http://creativecommons.org/licenses/by/4.0/

\begin{abstract}
Background: Anticipating postoperative evolution in surgical patients is an important issue in our daily practice. We demonstrated in a previous study that there were multiple predictors of postoperative outcome, including American Society of Anesthesiologists status (ASA), transfusion, emergency, surgery and age. A secondary analysis describing intraoperative and postoperative outcomes was undertaken in children aged between 6 and 10 years old included in the initial study. Objective: To describe intraoperative and postoperative outcomes in children aged between 6 and 10 years old included in the initial cohort in abdominal surgery, neurosurgery and orthopedics. Methods: The secondary analysis of postoperative outcomes in children aged between 6 and 10 years old was retrospectively included in the initial study of 594 patients. The study was approved by the Ethics Committee. Results: There were 88 patients with a mean age of $98.7 \pm 13.8$ months. The most common surgical interventions were scoliosis in 23 patients (26.1\%), limb tumor resection in 8 patients (9.1\%), femoral osteotomy in 6 patients $(6.8 \%)$, intracerebral tumor resection in 6 patients $(6.8 \%)$, intestinal resection in 5 patients (5.6\%), Chiari's malformation in 4 patients (4.5\%), pelvic osteotomy in 4 patients (4.5\%) and renal transplantation in 4 patients (4.5\%). Most patients (45\%) were American Society of Anesthesiologists grade 3 (ASA 3), and 13 (14.8\%) were ASA grade 4. Twenty-two (25\%) patients had intraoperative and/or postoperative complications (organ dysfunction or sepsis). Two patients $(2.3 \%)$ had intraoperative hemorrhage, 1 patient (1.1\%) had intraoperative difficult intubation, and 1 patient experienced intraoperative anaphylaxis. Nine patients $(10.2 \%)$ had postoperative neurologic failure, and $2(2.3 \%)$ had postoperative cardio-circulatory failure. Three patients (3.4\%) had postoperative septicemia, 2 patients $(2.3 \%)$ had postoperative pulmonary and urinary
\end{abstract}


sepsis, and 1 patient (1.1\%) had postoperative abdominal sepsis. 3 patients (3.4\%) had re-operations. 42 (47.7\%) patients had intra-operative transfusion. There was 1 in-hospital death (1.1\%). The median total length of hospital stay was 9 days [5 - 16]. Conclusion: Twenty-five percent of the patients had intraoperative and/or postoperative complications, and most of them were ASA grade $\geq 3$. Integrating goal-directed therapies to optimize intraoperative management in these patients could be necessary to improve postoperative outcomes in surgical pediatric patients.

\section{Keywords}

Children, Abdominal Surgery, Neurosurgery, Orthopedics, Outcome, Goal-Directed Therapies

\section{Introduction}

Postoperative outcomes in surgical patients are an important issue in our daily practice.

Predictors of postoperative outcome are multifactorial, among which American Society of Anesthesiologists status (ASA), transfusion, emergency, surgery and age were identified in previous studies [1] [2] [3] [4]. The ASA score (I-V) is a scale used in anesthesia to assess patient severity physical status: ASA I: normal healthy patient, ASA II: patient with mild systemic disease, ASA III: patient with severe systemic disease, ASA IV: patient with a severe systemic disease which is constantly threatening life, ASA V: the moribund patient who is not expected to survive without surgery.

Predictors of postoperative outcome in this study were not exhaustive, which means that other nonidentified factors may contribute to how patients evolve after surgery.

For a better postoperative outcome, anticipating patient management optimization begins preoperatively and continues intraoperatively and postoperatively. Intraoperative patient optimization includes fluid and hemodynamic goal-directed therapy with tools validated in children, blood patient transfusion protocols guided with point-of-care tests in hemorrhagic surgery and enhanced recovery after surgery protocols [5]-[13]. These goal-directed therapies have been shown in adults to improve postoperative outcomes [14]. In children, goal-directed therapies are not well developed and are not in routine generalized practice.

The study presented here had the objective of describing postoperative outcomes in children aged between 6 and 10 years who were included in the initial retrospective study [1]. The aim was to emphasize how these patients evolved after major surgery and to propose improvement implementation protocols.

\section{Methods and Materials}

A secondary analysis of children between 6 and 10 years old was included in the 
initial study [1].

The study was declared to the CNIL, National Commission for Computer Science and Liberties on 21 February 2017 under the registration number 2028257 v0. The Ethics Committee of Necker approved the study on 21 March 2017 under registration number 2017-CK-5-R1. Patients were included retrospectively from 1 January 2014 to 17 May 2017.

Figure 1 illustrates the inclusion and exclusion criteria flow chart.

The inclusion criteria were children aged between 6 and 10 years old.

The exclusion criteria were children aged less than 6 years old and older than 10 years.

Statistics were analyzed with XLSTAT 2020.4.1 software.

Continuous variables were described as the means \pm standard deviation or medians with interquartile ranges. Category variables were described in proportions.

\section{Results}

Table 1 illustrates the general characteristics.

There were 88 patients with a mean age of $98.7 \pm 13.8$ months.

There were 17 patients (19.3\%) who underwent abdominal surgery, 26 (29.5\%) who underwent neurosurgery and 45 (51.1\%) who underwent orthopedic surgery. 11 patients (12.5\%) had an emergency intervention.

Table 2 illustrates types of surgery.

The most common surgical interventions were scoliosis in 23 patients (26.1\%), limb tumor resection in 8 patients (9.1\%), femoral osteotomy in 6 patients (6.8\%),

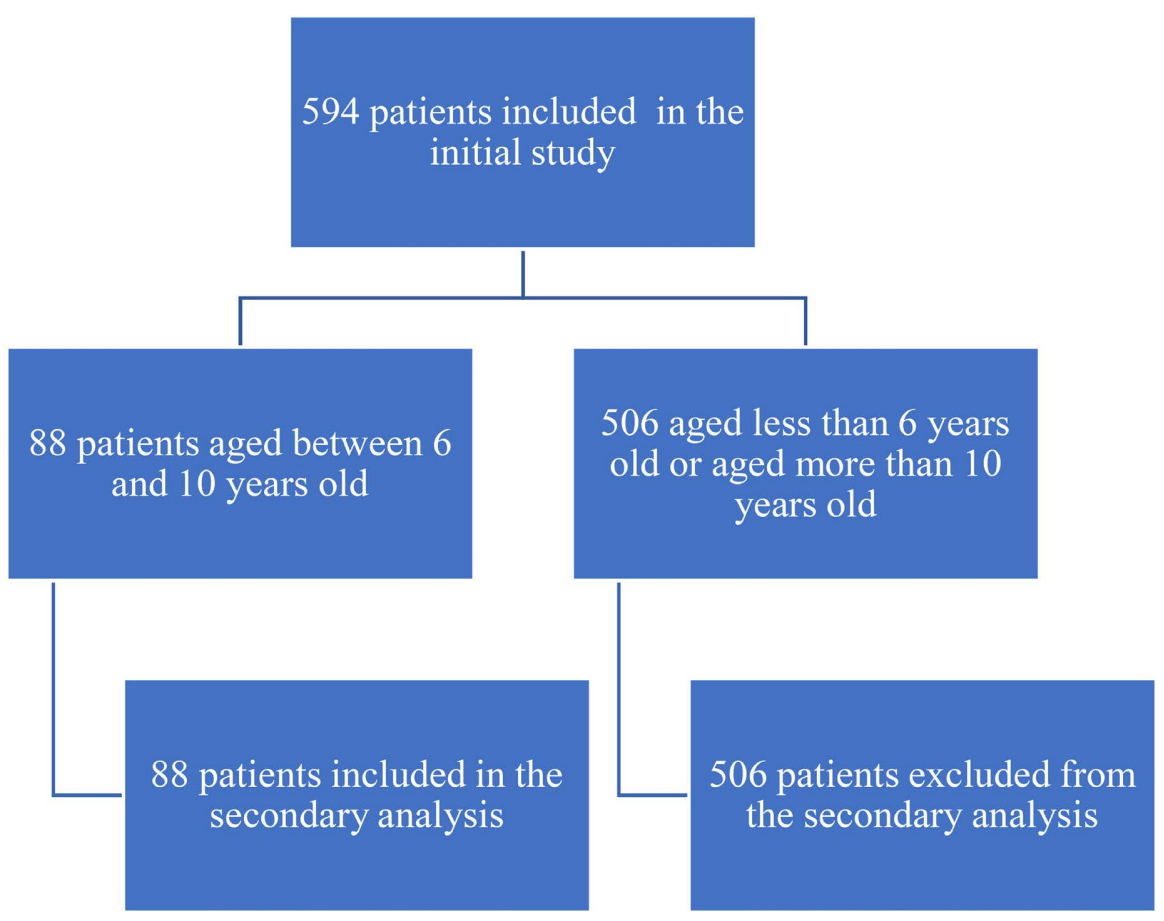

Figure 1. Inclusion and exclusion criteria flow chart. 
Table 1. General characteristics.

\begin{tabular}{|c|c|}
\hline Characteristics & $\mathrm{N}=88$ \\
\hline Mean age in months \pm standard deviation & $98.7 \pm 13.8$ \\
\hline Abdominal surgery $\mathrm{n}(\%)$ & $17(19.3)$ \\
\hline Neurosurgery n (\%) & $26(29.5)$ \\
\hline Orthopedic surgery n (\%) & $45(51.1)$ \\
\hline Elective surgery n (\%) & $77(87.5)$ \\
\hline Emergency surgery n (\%) & $11(12.5)$ \\
\hline Re-surgery n (\%) & $3(3.4)$ \\
\hline $\begin{array}{l}\text { Patients with intra-operative and or postoperative } \\
\text { complications (organ failure and or sepsis) } \mathrm{n}(\%)\end{array}$ & $22(25)$ \\
\hline Intra-operative hemorrhagic shock n (\%) & $2(2.3)$ \\
\hline Intra-operative difficult intubation n (\%) & $1(1.1)$ \\
\hline Intra-operative anaphylaxis n (\%) & $1(1.1)$ \\
\hline Postoperative neurologic failure n (\%) & $9(10.2)$ \\
\hline Postoperative cardio-circulatory failure n (\%) & $2(2.3)$ \\
\hline Postoperative endocrinal failure n (\%) & $1(1.1)$ \\
\hline Postoperative miscellaneous n (\%) & $1(1.1)$ \\
\hline Postoperative multi-organ failure n (\%) & $1(1.1)$ \\
\hline Postoperative septicemia n (\%) & $3(3.4)$ \\
\hline Postoperative pulmonary sepsis n (\%) & $2(2.3)$ \\
\hline Postoperative urinary sepsis n (\%) & $2(2.3)$ \\
\hline Postoperative abdominal sepsis n (\%) & $1(1.1)$ \\
\hline In hospital Mortality n (\%) & $1(1.1)$ \\
\hline Transfusion $\mathrm{n}(\%)$ & $42(47.7)$ \\
\hline Mean preoperative hemoglobin levels \pm standard deviation $\mathrm{g} / \mathrm{dL}$ & $11.6 \pm 1.9$ \\
\hline Mean postoperative hemoglobin levels \pm standard deviation $\mathrm{g} / \mathrm{dL}$ & $10.9 \pm 1.5$ \\
\hline ASA I n (\%) & $3(3.4)$ \\
\hline ASA II n (\%) & $31(35.2)$ \\
\hline ASA III n (\%) & $40(45.5)$ \\
\hline ASA IV n (\%) & $13(14.8)$ \\
\hline ASA V n $(\%)$ & $1(1.1)$ \\
\hline Median length of intensive care unit stay in days [interquartile range] & $4[1-6]$ \\
\hline Median length of hospital stay in days [interquartile range] & $6[3.7-10.2]$ \\
\hline Median total length of hospital stay in days [interquartile range] & $9[5-16]$ \\
\hline $\begin{array}{l}\text { Median length of mechanical ventilation (invasive or non-invasive) in } \\
\text { days [interquartile range] }\end{array}$ & $0[0-1]$ \\
\hline
\end{tabular}


Table 2. Surgery.

\begin{tabular}{|c|c|}
\hline Surgery & Number of patients (\%) \\
\hline Basal skull schwannoma & $3(3.4)$ \\
\hline Brainstem lesion resection & $1(1.1)$ \\
\hline $\begin{array}{l}\text { Cerebral aneurysm/Cerebral arterio-venous } \\
\text { malformation angioembolization }\end{array}$ & $1(1.1)$ \\
\hline Cerebral cavernoma & $1(1.1)$ \\
\hline Chiari's malformation & $4(4.5)$ \\
\hline Craniosynostosis & $3(3.4)$ \\
\hline Epileptogenic lesion resection & $1(1.1)$ \\
\hline Femoral osteotomy & $6(6.8)$ \\
\hline Femoral prothesis & $1(1.1)$ \\
\hline Hepatic tumor & $1(1.1)$ \\
\hline Interscapular thoracic desarticulation & $2(2.2)$ \\
\hline Intestinal resection & $5(5.6)$ \\
\hline Intracerebral tumor resection & $6(6.8)$ \\
\hline Knee prothesis & $1(1.1)$ \\
\hline Laparotomy for volvulus & $1(1.1)$ \\
\hline Limb tumor resection & $8(9.1)$ \\
\hline Liver transplantation & $3(3.4)$ \\
\hline Neuroblastoma & $1(1.1)$ \\
\hline Orbital tumor & $1(1.1)$ \\
\hline Pelvic osteotomy & $4(4.5)$ \\
\hline Pelvic tumor & $1(1.1)$ \\
\hline Posterior fossa decompression & $1(1.1)$ \\
\hline Renal transplantation & $4(4.5)$ \\
\hline Scoliosis & $23(26.1)$ \\
\hline Splenectomy & $1(1.1)$ \\
\hline Ventriculostomy & $1(1.1)$ \\
\hline Vertebral laminectomy/Arthrodesis & $3(3.4)$ \\
\hline
\end{tabular}

intracerebral tumor resection in 6 patients $(6.8 \%)$, intestinal resection in 5 patients (5.6\%), Chiari's malformation in 4 patients (4.5\%), pelvic osteotomy in 4 patients (4.5\%) and renal transplantation in 4 patients (4.5\%).

Most patients (45\%) were ASA grade 3, and 13 (14.8\%) patients were ASA grade 4 .

Twenty-two (25\%) patients had intraoperative and/or postoperative complications (organ dysfunction or sepsis). 
The most common intraoperative complication was hemorrhagic shock in 2 patients $(2.3 \%)$, followed by difficult intubation and anaphylaxis in 1 patient (1.1\%). The most common postoperative organ failure was neurologic in 9 patients $(10.2 \%)$, followed by cardio-circulatory in 2 patients $(2.3 \%)$. The most common postoperative infection was septicemia in 3 patients (3.4\%), followed by pulmonary and urinary sepsis in 2 patients $(2.3 \%)$ and abdominal sepsis in 1 patient (1.1\%). 3 patients (3.4\%) had re-operations. $42(47.7 \%)$ patients had intra-operative transfusion. There was 1 in-hospital death (1.1\%) (Table 3 ).

The median total length of hospital stay was 9 days [5 - 16].

Table 4 illustrates outcomes per surgery.

Table 5 illustrates co-morbidities. The most common comorbidities were intracerebral tumor in 7 patients (7.9\%), Ewing's sarcoma in 5 patients (5.7\%), psychomotor deficiency in 5 patients (5.7\%), arthritis in 4 patients (4.5\%), cerebral anoxic lesions in 4 patients (4.5\%), chronic renal failure in 4 patients $(4.5 \%)$, hepatic failure in 4 patients (4.5\%) and polymalformation syndrome in 4 patients (4.5\%).

\section{Discussion and Conclusion}

The rate of patients with intraoperative and/or postoperative complications in this cohort of 88 children between 6 and 10 years of age who underwent major abdominal surgery, neurosurgery and orthopedics was $25 \%$. These patients were in the majority ASA grade 3 or more. As revealed in the initial studies [1] [2] [3] [4], the postoperative outcome depends on multiple factors, precisely ASA status, transfusion, age, emergency and surgery. Integrating goal-directed therapies for intraoperative management in these patients could be necessary to improve postoperative outcomes in pediatric surgical patients. Goal-directed therapies include intraoperative fluid and hemodynamic goal-directed therapy with validated tools in children, intraoperative transfusion goal-directed protocols with point-of-care devices to guide blood product administration and enhanced recovery after surgery [5]-[13]. All these therapies have the same aim, which is to optimize the relation between oxygen consumption and oxygen delivery [6] [7]. Optimization of oxygen consumption-oxygen delivery relation has the goal of improving intraoperative patient status that contributes to a favorable postoperative evolution [6] [7]. In our hospital, goal-directed therapies are not yet routine generalized practice. It is time to reconsider integrating goal-directed therapies

Table 3. Patient with fatal outcome.

\begin{tabular}{ccccccccc}
\hline Surgery & $\begin{array}{c}\text { Age } \\
\text { months }\end{array}$ & $\begin{array}{c}\text { ASA } \\
\text { score }\end{array}$ & Co-morbidities & $\begin{array}{c}\text { Intra-operative } \\
\text { complications }\end{array}$ & $\begin{array}{c}\text { Postoperative } \\
\text { outcome }\end{array}$ & $\begin{array}{c}\text { Delay of } \\
\text { in-hospital } \\
\text { mortality in } \\
\text { days }\end{array}$ \\
\hline $\begin{array}{c}\text { Posterior fossa } \\
\text { decompression }\end{array}$ & 85 & 5 & Sickle cell disease & 0 & $\begin{array}{c}\text { Multiple organ } \\
\text { failure and } \\
\text { pulmonary sepsis }\end{array}$ & 7 & Yes Transfusion \\
\hline
\end{tabular}


Table 4. Outcomes per surgery.

Surgery

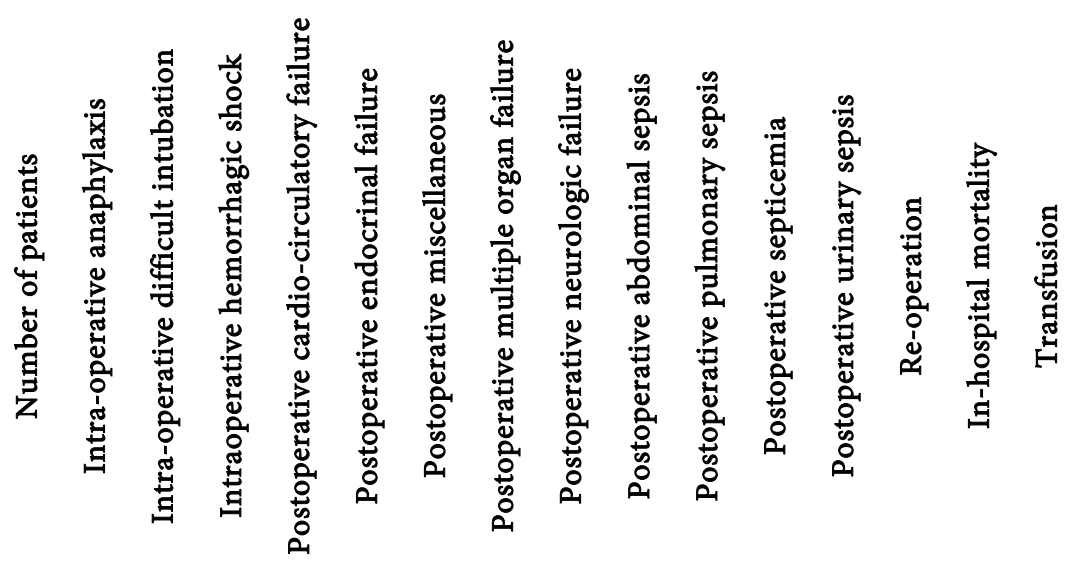

Basal skull schwannoma

$\begin{array}{llllllllllllllll}3 & 0 & 0 & 0 & 0 & 0 & 0 & 0 & 0 & 0 & 0 & 0 & 0 & 0 & 0 & 1\end{array}$

Brainstem lesion resection

Cerebral aneurysm/Cerebral arterio-venous malformation angioembolization

Cerebral cavernoma

Chiari's malformation

Craniosynostosis

Epileptogenic lesion resection

Femoral osteotomy

Femoral prothesis

Hepatic tumor

Interscapular thoracic desarticulation

Intestinal resection

Intracerebral tumor resection

Knee prothesis

Laparotomy for volvulus

Limb tumor resection

Liver transplantation

Neuroblastoma

Orbital tumor

Pelvic osteotomy

Pelvic tumor

Posterior fossa decompression

Renal transplantation

Scoliosis

Splenectomy

Ventriculostomy

Vertebral laminectomy/Arthrodesis

$\begin{array}{llllllllllllllll}1 & 0 & 0 & 0 & 0 & 0 & 0 & 0 & 0 & 0 & 0 & 0 & 0 & 0 & 0 & 1\end{array}$

$\begin{array}{llllllllllllllll}1 & 0 & 0 & 0 & 0 & 0 & 0 & 0 & 1 & 0 & 0 & 0 & 1 & 0 & 0 & 1\end{array}$

$\begin{array}{llllllllllllllll}1 & 0 & 0 & 0 & 0 & 0 & 0 & 0 & 0 & 0 & 0 & 0 & 0 & 0 & 0 & 0\end{array}$

$\begin{array}{llllllllllllllll}4 & 0 & 0 & 0 & 0 & 0 & 0 & 0 & 0 & 0 & 0 & 0 & 0 & 0 & 0 & 0\end{array}$

$\begin{array}{llllllllllllllll}3 & 0 & 0 & 0 & 0 & 0 & 0 & 0 & 1 & 0 & 0 & 0 & 0 & 0 & 0 & 1\end{array}$

$\begin{array}{llllllllllllllll}1 & 0 & 0 & 0 & 0 & 0 & 0 & 0 & 1 & 0 & 0 & 0 & 0 & 0 & 0 & 1\end{array}$

$\begin{array}{llllllllllllllll}6 & 0 & 0 & 1 & 0 & 0 & 0 & 0 & 1 & 0 & 0 & 0 & 0 & 0 & 0 & 3\end{array}$

$\begin{array}{llllllllllllllll}1 & 0 & 0 & 0 & 0 & 0 & 0 & 0 & 0 & 0 & 0 & 0 & 0 & 0 & 0 & 1\end{array}$

$\begin{array}{llllllllllllllll}1 & 0 & 0 & 0 & 0 & 0 & 0 & 0 & 0 & 0 & 0 & 0 & 0 & 0 & 0 & 1\end{array}$

$\begin{array}{llllllllllllllll}2 & 0 & 0 & 0 & 0 & 0 & 0 & 0 & 0 & 0 & 0 & 0 & 0 & 0 & 0 & 2\end{array}$

$\begin{array}{llllllllllllllll}5 & 0 & 0 & 0 & 0 & 0 & 0 & 0 & 0 & 0 & 0 & 0 & 0 & 0 & 0 & 0\end{array}$

$\begin{array}{llllllllllllllll}6 & 1 & 0 & 0 & 0 & 1 & 0 & 0 & 0 & 0 & 0 & 0 & 0 & 0 & 0 & 1\end{array}$

$\begin{array}{llllllllllllllll}1 & 0 & 0 & 0 & 0 & 0 & 0 & 0 & 0 & 0 & 0 & 0 & 0 & 0 & 0 & 1\end{array}$

$\begin{array}{llllllllllllllll}1 & 0 & 0 & 0 & 0 & 0 & 0 & 0 & 0 & 0 & 0 & 0 & 0 & 0 & 0 & 1\end{array}$

$\begin{array}{llllllllllllllll}8 & 0 & 0 & 0 & 0 & 0 & 0 & 0 & 0 & 0 & 0 & 0 & 0 & 0 & 0 & 4\end{array}$

$\begin{array}{llllllllllllllll}3 & 0 & 0 & 0 & 1 & 0 & 1 & 0 & 0 & 1 & 0 & 0 & 0 & 1 & 0 & 3\end{array}$

$\begin{array}{llllllllllllllll}1 & 0 & 1 & 0 & 0 & 0 & 0 & 0 & 0 & 0 & 0 & 0 & 0 & 0 & 0 & 1\end{array}$

$\begin{array}{llllllllllllllll}1 & 0 & 0 & 0 & 0 & 0 & 0 & 0 & 0 & 0 & 0 & 0 & 0 & 0 & 0 & 0\end{array}$

$\begin{array}{llllllllllllllll}4 & 0 & 0 & 0 & 0 & 0 & 0 & 0 & 0 & 0 & 0 & 0 & 0 & 0 & 0 & 3\end{array}$

$\begin{array}{llllllllllllllll}1 & 0 & 0 & 0 & 0 & 0 & 0 & 0 & 0 & 0 & 0 & 0 & 0 & 0 & 0 & 0\end{array}$

$\begin{array}{llllllllllllllll}1 & 0 & 0 & 0 & 0 & 0 & 0 & 1 & 0 & 0 & 1 & 0 & 0 & 0 & 1 & 1\end{array}$

$\begin{array}{llllllllllllllll}4 & 0 & 0 & 0 & 0 & 0 & 0 & 0 & 0 & 0 & 0 & 0 & 1 & 0 & 0 & 2\end{array}$

$\begin{array}{llllllllllllllll}23 & 0 & 0 & 1 & 1 & 0 & 0 & 0 & 3 & 0 & 0 & 2 & 0 & 1 & 0 & 9\end{array}$

$\begin{array}{llllllllllllllll}1 & 0 & 0 & 0 & 0 & 0 & 0 & 0 & 0 & 0 & 0 & 0 & 0 & 0 & 0 & 1\end{array}$

$\begin{array}{llllllllllllllll}1 & 0 & 0 & 0 & 0 & 0 & 0 & 0 & 1 & 0 & 1 & 0 & 0 & 0 & 0 & 1\end{array}$

$\begin{array}{llllllllllllllll}3 & 0 & 0 & 0 & 0 & 0 & 0 & 0 & 1 & 0 & 0 & 1 & 0 & 1 & 0 & 2\end{array}$ 
Table 5. Co-morbidities.

Co-morbidity

Arachnoid cyst

Arthritis

Bronchodysplasia sequelae

Cancer

Cerebral aneurysm/Cerebral arterio-venous

malformation, Pulmonary hypertension

Cerebral anoxic lesions

Chiari's malformation

Chronic renal failure

$4(4.5)$

Convulsive encephalopathy

$1(1.1)$

Crohn's disease

$1(1.1)$

Epilepsy

$2(2.2)$

Ewing's sarcoma

Extradural hematoma

Gorlin's syndrome

$1(1.1)$

Hepatic failure

$4(4.5)$

Intestinal pseudo-occlusion

$1(1.1)$

Intracerebral tumor

7 (7.9)

Klippel-Feil Syndrome

$1(1.1)$

Larsen syndrome

$1(1.1)$

Morquio syndrome

$1(1.1)$

Myelomeningocele

Necrotizing enterocolitis sequelae

$1(1.1)$

Neurofibromatosis

$3(3.4)$

Pierre Robin syndrome

$1(1.1)$

Polymalformation syndrome

$4(4.5)$

Polytrauma

$2(2.2)$

Psychomotor deficiency

$5(5.7)$

Rachitism

$1(1.1)$

Sarcoidosis

Sickle cell disease

$1(1.1)$

Crouzon syndrome

$1(1.1)$

in intraoperative patient management in high-risk patients and surgery to improve postoperative outcomes. 


\section{Author's Contributions}

Claudine Kumba conceptualized and designed the study and drafted the initial manuscript. She designed the data collection instruments, collected data, carried out initial and final analyses.

\section{Conflicts of Interest}

The author declared no conflicts of interest regarding the publication of this paper.

\section{References}

[1] Kumba, C., Cresci, F., Picard, C. et al. (2017) Transfusion and Morbi-Mortality Factors: An Observational Descriptive Retrospective Pediatric Cohort Study. Journal of Anesthesia \& Critical Care Open Access, 8, Article No. 00315. https://doi.org/10.15406/jaccoa.2017.08.00315

[2] Kumba, C., Taright, H., Terzi, E., Telion, C., Beccaria, K., Paternoster, G., Zerah, M., Bustarret, O., Jugie, M., Rubinsztajn, R. and Treluyer, J.M. (2018) Blood Product Transfusion and Postoperative Outcome in Pediatric Neurosurgical Patients. EC Anaesthesia, 4, 288-298.

[3] Kumba, C., Lenoire, A., Cairet, P., Dogaru-Dedieu, E., Belloni, I. and Orliaguet, G. (2018) Is Transfusion an Independent Risk Factor of Postoperative Outcome in Pediatric Orthopedic Surgical Patients? A Retrospective Study. Journal of Emergency Medicine \& Critical Care, 4, Article No. 7. https://doi.org/10.13188/2469-4045.1000018

[4] Kumba, C., Querciagrossa, S., Blanc, T. and Treluyer, J.M. (2018) Transfusion and Postoperative Outcome in Pediatric Abdominal Surgery. Journal of Clinical Research in Anesthesiology, 1, 1-8. https://doi.org/10.33309/2639-8915.010103

[5] Kumba, C. (2019) Do Goal Directed Therapies Improve Postoperative Outcome in Children? (Perioperative Goal Directed Fluid and Hemodynamic Therapy; Transfusion Goal Directed Therapy Using Viscoelastic Methods and Enhanced Recovery after Surgery and Postoperative Outcome): A Study Research Protocol. Acta Scientific Paediatrics, 2, 17-19. https://doi.org/10.31080/ASPE.2019.02.0094

[6] Kumba, C. (2020) Physiology Principles Underlying Goal Directed Therapies in Children. Research in Pediatrics \& Neonatology, 4, RPN.000591.

https://doi.org/10.31031/RPN.2020.04.000591

[7] Kumba, C. (2020) Rationale of Goal Directed Therapies in Children. Advances in Pediatric Research, 7, Article No. 42.

[8] Kumba, C., Willems, A., Querciagrosssa, S., Harte, C., Blanc, T., et al. (2019) A Systematic Review and Meta-Analysis of Intraoperative Goal Directed Fluid and Haemodynamic Therapy in Children and Postoperative Outcome. Journal of Emergency Medicine \& Critical Care, 5, Article No. 9. https://doi.org/10.13188/2469-4045.1000020

[9] Kumba, C. (2020) Goal Directed Fluid and Hemodynamic Therapy and Postoperative Outcomes in Children: Value of Transthoracic Echocardiographic Aortic Blood Flow Peak Velocity Variation: A Multi-Centre Randomized Controlled Trial Protocol. dvances in Pediatric Research, 7, Article No. 35. https://doi.org/10.35248/2385-4529.20.7.35

[10] Kumba, C. (2020) Trans-Thoracic Echocardiographic Aortic Blood Flow Peak Ve- 
locity Variation, Distance Minute, Aortic Velocity Time Integral and Postoperative Outcome in Pediatric Surgical Patients-An Observational Pilot Study Protocol. Open Journal of Internal Medicine, 10, 90-95.

https://doi.org/10.4236/ojim.2020.101009

[11] Kumba, C., Querciagrossa, S., Harte, C., Willems, A., De Cock, A., Blanc, T., et al. (2019) A Systematic Review and Meta-analysis of Goal Directed Intra-Operative Transfusion Protocols Guided by Viscoelastic Methods and Perioperative Outcomes in Children. International Journal of Recent Scientific Research, 10, 31466-31471.

[12] Kumba, C., Blanc, T., De Cock, A., Willems, A., Harte, C., Querciagrossa, S., et al. (2019) Rapid Recovery Pathways after Surgery in Children: A Systematic Review and Meta-Analysis. Medical Journal of Clinical Trials \& Case Studies, 3, Article No. 000211.

[13] Kumba, C. and Melot, C. (2019) The Era of Goal Directed Therapies in Paediatric Anaesthesia and Critical Care. EC Emergency Medicine and Critical Care, 3, No, 5, 306-309.

[14] Chong, M., Wang, Y., Berbenetz, N., et al. (2018) Does Doal-Directed Haemody-Namic and Fluid Therapy Improve Peri-Operative Outcomes? A Systematic Review and Meta-Analysis. European Journal of Anaesthesiology, 35, 469-483.

https://doi.org/10.1097/EJA.0000000000000778 